\title{
Caracterización de las Diatomitas de Río Seco (Piura, Perú)
}

\author{
J. RODRíGUEZ LICHTENHELDT $(*)$, J. LOREDO PÉREZ $(* *)$, L. FELIPE VERDEJA $(* *)$, J. GARCíA IGLESIAS $(* *)$ y J. SANCHO MARTíNEZ (**). \\ (*) Facultad de Minas. Universidad Nacional de Piura. Perú. (**) E.T.S. Ingenieros de Minas. Universidad de Oviedo. España.
}

\begin{abstract}
Resumen: La existencia de diatomita en la zona de Río Seco (Piura, Perú), ha llevado a los autores a realizar un estudio de sus propiedades físicas, químicas y mineralógicas, con el objeto de determinar sus principales usos y aplicaciones. Para ello se han utilizado las técnicas de análisis convencional, así como microscopía óptica y electrónica, difracción de rayos X y análisis térmico diferencial y gravimétrico. Tratándose de un material de calidad media, por los contenidos en sílice y alúmina que presenta, su aplicación quedaría restringida a la fabricación de materiales puzolánicos y cerámicos para la construcción.
\end{abstract}

Palabras clave: Diatomita, Caracterización, Perú.

\section{Characterization of Diatomitas form Rio Seco (Piura, Peru)}

Abstract: The occurrence of diatomites on the area of Río Seco (Piura, Peru), has led the authors to study their physical, chemical and mineralogical properties with the aim to determinate their principal uses and applications. Conventional methods were applied besides of optical and electronical microscopy, difraction of $X$ rays and thermal differential and gravimetric analysis. It is a medium quality material because of their silica and alumina contents, then the applications should be limited to fabrication of puzzolanic and ceramic materials for construction.

Key words: Diatomite, Characterisation, Peru.

\section{INTRODUCCIÓN.}

El presente trabajo se enmarca dentro de una línea de investigación iniciada en el año 1.988 por la E.T.S. de Ingenieros de Minas de Oviedo (Departamentos de "Ciencias de los Materiales e Ingeniería Metalúrgica" y de "Explotación y Prospección de Minas"), en colaboración con la Universidad Nacional de Piura, el Instituto para la Promoción de la Minería no Metálica (NMI) de Lima, y la Universidad de Piura, para el estudio, caracterización y aplicaciones de los recursos mineros no metálicos del noroeste peruano (1) (2) (3) y (4).

La diatomita es una roca que procede de la acumulación de caparazones silíceos de organismos unicelulares denominados diatomeas. Éstas, son algas unicelulares integrables dentro de la clase Bacillario-phyceae, que habitan en la zona fótica de océanos y lagos, con concentraciones en las aguas que pueden variar desde unos pocos millares a varios millones de individuos por litro (5). Las diatomeas típicas presentan formas circulares (céntricas) o elípticas (pinadas). La clasificación de las diatomeas se basa en aspectos morfológicos, a partir de los cuales se han establecido más de 300 géneros y entre 12.000 y 16.000 especies (6).

Estos organismos, cualesquiera que sea su género y especie, tienen la capacidad de extraer sílice de su hábitat natural, formando una estructura silícea denominada frústula, consistente en dos valvas finamente perforadas, que recubre la cara externa del protoplasma en un 10 a un $30 \%$ de su superficie (7). El diámetro de las frústulas oscila entre 5 y $2.000 \mu \mathrm{m}$, con una talla media más frecuente entre 20 y $200 \mu \mathrm{m}$; si bien el tamaño, forma y diseño de esta estructura son características de cada especie.

La diatomita se compone fundamentalmente de sílice amor$\mathrm{fa}$, con pequeñas cantidades de aluminio, hierro y metales alcalinos en forma de óxidos, así como de otros constituyentes menores como el titanio y cantidades variables de materia orgánica, sales solubles, arcillas, carbonatos, etc. En su estado natural, la diatomita tiene una cantidad inusual de agua, que puede variar entre un 10 y un $60 \%$.

La calidad del mineral crece con el porcentaje de sílice, y desciende al aumentar el contenido de los diferentes elementos ( $\mathrm{Fe}, \mathrm{Ca}, \mathrm{Na}, \mathrm{K}, \mathrm{Al}$, etc.), que son los que constituyen las impurezas de la diatomita. Para determinadas aplicaciones de la diatomita, el mercado establece límites en el contenido de estos componentes.

Las diatomitas presentan un aspecto de tiza, con color blanco, y densidad y dureza extremadamente bajas. Son muy porosas y fácilmente deleznables. Estos aspectos se reflejan en la estructura íntima de la roca, a la que le confieren una alta capacidad absorbente para los líquidos, una gran área superficial, conductividad térmica baja, capacidad abrasiva suave, así como ser químicamente inertes, lo que la hacen extraordinariamente indicada para determinadas aplicaciones en la industria, como material filtrante, material de relleno, aislante térmico, etc., para muchas de las cuales la diatomita ha demostrado ser un recurso mineral irremplazable.

El concepto de alto o bajo grado, referido a la calidad de las 
diatomitas, no depende solamente de sus características propias, sino también del uso al que van a ser destinadas; ya que dada la amplia variedad de aplicaciones, un tipo concreto de diatomita puede resultar efectivo para un determinado uso, pero puede tener escasa utilidad para otro. No obstante, tradicionalmente se ha considerado la diatomita de alta calidad a aquella que presenta los mayores contenidos en sílice (8).

La diatomita es una sustancia muy abundante en Perú, pudiendo destacar de entre sus yacimientos, los existentes en la Región Grau, situada al noroeste del país (1) (4) (9). Con este artículo se pretende aportar datos sobre las características físicas, químicas y mineralógicas de la diatomita de uno de los yacimientos minerales existentes en la zona de Piura, concretamente el ubicado en la localidad de "Río Seco", próximo a dicha ciudad, con el fin de definir posibles campos de aplicación. Hasta ahora, las diatomitas de "Río Seco" se han venido utilizando de forma local, con muy buen resultado para la fabricación artesanal de ladrillos y cubiertas para techos.

\section{LOCALIZACIÓN Y DESCRIPCIÓN DEL YACIMIENTO.}

El área en la que se encuentra el yacimiento de diatomita de "Río Seco", se ubica en la zona noroccidental del Perú, y está limitada al este y al sur por el desierto de Sechura, correspondiente a la cuenca terciaria del mismo nombre, la cual se formó a partir de una tectónica de bloques que dio origen a una secuencia de horst y graben a lo largo de la zona norte de Perú; en ella los horst se corresponden con rocas metamórficas e ígneas precámbricas, paleozoicas y cretáceas, y los graben están rellenos por sedimentos terciarios y cuaternarios.

Esta zona -la cuenca de Sechura- pertenece a la Depresión Para-Andina, que es una unidad geomorfológica extendida a lo largo de todo el noroeste peruano, entre la Cordillera de la Costa y los contrafuertes de la Cordillera Occidental, siguiendo un lineamiento paralelo a la Cordillera de los Andes, y se caracteriza por estar formada casi exclusivamente por sedimentos correspondientes al terciario, representado en la región por materiales que van del Eoceno Superior al Mioceno Inferior, y cuaternarios (10).

Dentro de la Región Grau, el yacimiento se encuentra localizado en la zona conocida como Pampa Tierra Blanca, en el paraje denominado Río Seco, a unos $7 \mathrm{~km}$. al noroeste de la ciudad de Piura.

La diatomita se encuentra incluida en la formación Zapallal, correspondiente al Mioceno Superior, que en la zona del yacimiento presenta una potencia de unos 120 metros, con un ligero recubrimiento de sedimentos cuaternarios. La litología de la formación es muy característica: El tramo superior está constituido por areniscas de grano fino, interestratificadas con lutitas, limolitas y bentonitas, en su tramo medio aparecen areniscas conglomeráticas, y el tramo inferior se caracteriza por sus horizontes arcillosos, que se intercalan con paquetes de fosfatos (11)

Las diatomitas se encuentran formando capas dentro de la parte alta del tramo superior. En el área de estudio, tenemos un nivel bien caracterizado que, cuando aflora, resulta fácilmente erosionable y visible en amplias zonas, o bien con recubrimiento de materiales eólicos cuaternarios que alcanzan unos $30 \mathrm{~cm}$. de potencia, como término medio, en el resto del área.

Se trata de un depósito mineral cuya dimensión mayor sigue una dirección N-S, paralela al cauce del río Piura, y que se extiende con una disposición horizontal a lo largo de una superficie de unos $10 \mathrm{~km}$. de largo por $1 \mathrm{~km}$. de ancho, con una potencia comprendida entre 0,5 y $2,5 \mu$. Las reservas estimadas de diatomita se evalúan en unos cuatro millones de toneladas (12). Estamos por tanto, ante un yacimiento con condiciones óptimas para su explotación a cielo abierto y con comunicaciones ya establecidas.

\section{CARACTERÍSTICAS FÍSICAS, QUÍMICAS Y MINERALÓGICAS.}

Para la caracterización físico-química de las diatomitas del yacimiento de "Río Seco", se han utilizado 14 muestras tomadas en zonas diferentes del yacimiento y representativas del mismo. Aún cuando el número de muestras utilizadas para la caracterización del material no es muy grande, dada la uniformidad de las características visibles del yacimiento y la constancia de los datos físico-químicos que presentan las muestras estudiadas, se puede considerar suficiente para una caracterización inicial de estos niveles de diatomitas.

\subsection{Caracterización física.}

Previamente a la determinación de las características físicas del material, se ha realizado un estudio morfológico sobre muestras representativas de diatomita, para determinar las microestructuras que presentan las diatomeas; para ello se ha utilizado microscopía electrónica de barrido (SEM), con un microscopio JEOL 2000. Se observa un predominio de morfologías de tipo acicular con largas frústulas y morfologías de tipo cilíndrica y curvilínea (figura 1). También han sido identificadas otras morfologías de diatomeas con valvas circulares fuertemente convexas. La presencia de esponjas y espículas silíceas es también abundante.

En la estructura primaria de las diatomeas, referida a orificios o poros de las paredes, se determinó un tamaño promedio de poro entre 0,5 y $1 \mu$. También existen otros orificios en la

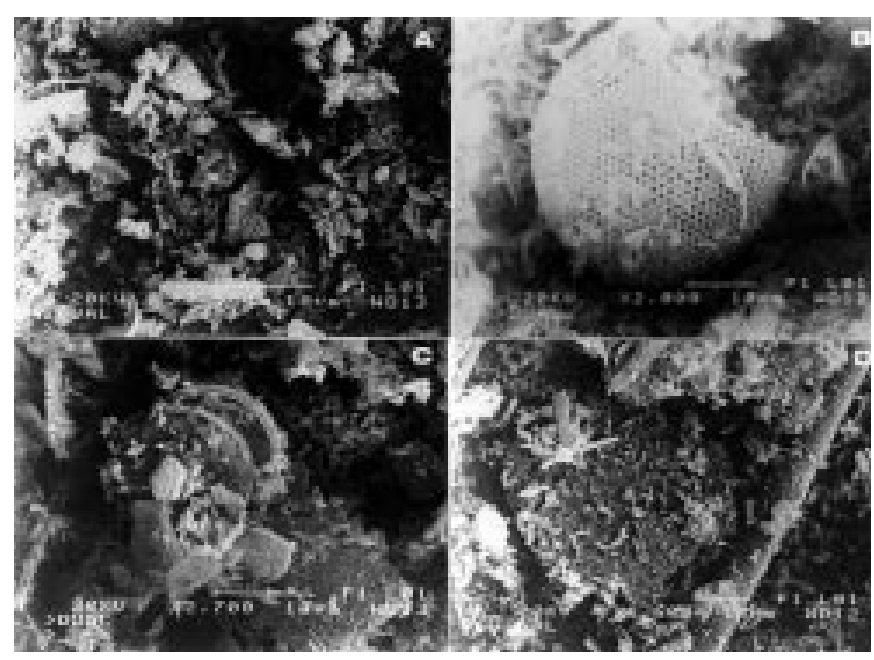

Fig. 1. Microfotografías mostrando diferentes aspectos de la diatomita de "Río Seco".
A) Aspecto general de la diatomita.
B) Caparazón de diatomea central.
C) Diatomea central en la que se observan las dos valvas.
D) Diatomea acicular en borde de diatomea central. 
misma cámara, que representan tanto la estructura secundaria como terciaria, que en la valva de la diatomea llegan a tener dimensiones del orden de micras.

Para la caracterización física de la diatomita se han medido las siguientes propiedades:

- Densidad global (DG) (Micrometrics Pore Sizer 9350, Hg).

- Densidad real (DR) (Micrometrics Autopycnometer 1320, He).

- Contenido de agua (relación entre peso de muestra húmeda y peso de muestra deshidratada).

- Índice de poros (IP = DR/DG - 1).

- Porosidad total (relación entre volumen de huecos y volumen total de muestra).

- Porosidad abierta (Micrometrics Porosizer 9305, Hg).

- Superficie específica (Micrometrics Surface Analyzer 2200. $\mathrm{N}_{2}$ BET).

- Absorción en agua y aceite (Método Westinghouse).

- Índice de blancura (Fotómetro Carl Zeiss).

De los resultados obtenidos para las 14 muestras estudiadas (tabla I), se pueden deducir las siguientes características:

- La densidad real, con la excepción de la muestra número 8, se encuentra dentro de los rangos normales que presentan las diatomitas, a pesar de la existencia de un material arcilloso que actúa como contaminante. Los valores medidos varían entre un mínimo de $1,754 \mathrm{~g} / \mathrm{cm}^{3}$ para la muestra $\mathrm{M}$ 8 , y un máximo de $2,381 \mathrm{~g} / \mathrm{cm}^{3}$ para la muestra $\mathrm{M}-11$. El valor promedio de la densidad real es de $2,200 \mathrm{~g} / \mathrm{cm}^{3}$.

- El porcentaje del contenido de agua de las muestras es muy bajo, oscilando entre 2,51 y 7,74\%.

- Los rangos de porosidad total se hallan comprendidos entre 48 y $63 \%$, calificándose como un material de porosidad media a alta.

- La superficie específica BET es alta, encontrándose valores comprendidos entre 5,6 y $10,5 \mathrm{~m}^{2} / \mathrm{g}$.

- La absorción en agua y la absorción en aceite, los cuales son datos fundamentales para la utilización de la diatomita como material filtrante, indican valores comprendidos entre
90 y $134 \%$ para la absorción en agua, y entre 113 y $166 \%$ para la absorción en aceite.

\subsection{Caracterización química.}

El análisis químico de las muestras ha sido realizado por fluorescencia de rayos X, con un equipo Philips PW 1480. Los datos correspondientes al análisis químico de las 14 muestras estudiadas aparecen en la tabla II. El contenido promedio de sílice es de $67,1 \%$, lo que comparándola con la diatomita natural Foselite-24 (1), que tiene un contenido del $88,4 \%$ de óxido de sílice, permite clasificar a la diatomita de Río Seco como de calidad media.

Las impurezas se encuentran representadas por los siguientes elementos:

- El aluminio, cuyo valor en $\mathrm{Al}_{2} \mathrm{O}_{3}$ se sitúa entre $11,6 \%$ y $14,5 \%$, con un valor promedio de $17 \%$. Se puede considerar que su presencia es debida al contenido de arcillas y feldespatos de las muestras.

- Los metales alcalinos sodio y potasio presentan unos contenidos promedios de 3,3\% de $\mathrm{Na}_{2} \mathrm{O}$ y de $3,52 \%$ de $\mathrm{K}_{2} \mathrm{O}$ atribuyéndose a la presencia de feldespatos y cloruros, producto de la precipitación marina.

- Los metales alcalino-térreos calcio y magnesio presentan porcentajes de sus óxidos metálicos respectivos siempre inferiores al $2 \%$, excepto las muestras M-7 y M-8, que presentan contenidos en $\mathrm{CaO}$ de $8,8 \%$, como consecuencia de la presencia significativa de calcita en las mismas.

- El titanio presente en las muestras nunca supera el 0,31 \% en $\mathrm{TiO}_{2}$, y su valor promedio es $0,26 \%$.

- Dentro de los elementos traza, el más abundante es el bario, que muestra valores entre 505 y 989 ppm. Otros elementos con cantidades significativas son el Zr (149ppm-198ppm), Sr (210ppm-467ppm) y Rb (91ppm-155ppm).

El pH de las muestras se determinó en suspensiones al 5\%. Los resultados obtenidos indican datos muy uniformes para el conjunto de las 14 muestras estudiadas, a las que corresponden valores comprendidos entre 9,10 y 9,85.

TABLA I.

Propiedades físicas de la diatomita de "Río SeCo".

\begin{tabular}{|c|c|c|c|c|c|c|c|c|c|c|}
\hline MUESTRA & D.R.: $\mathrm{g} / \mathrm{cm} 3$ & D.G.: $\mathrm{g} / \mathrm{cm} 3$ & I.P. & P.T. (\%) & S.P.(\%) & P:A:/\%) & $\mathrm{AB} . \mathrm{H} 2 \mathrm{O}(\%)$ & AB. AC. $(\%)$ & S.E.:m2/g & $W(\%)$ \\
\hline 1 & 2,326 & 0,975 & 1,39 & 58 & 12 & 49 & 127 & 165 & 8,7 & 7,43 \\
\hline 2 & 2,273 & 0,904 & 1,51 & 60 & 12 & 42 & 105 & 141 & 7,7 & 7,74 \\
\hline 3 & 2,141 & 0,861 & 1,48 & 59 & 6 & 44 & 111 & 122 & 7,1 & 4,32 \\
\hline 5 & 2,501 & 0,989 & 1,52 & 60 & 7 & 46 & 138 & 147 & 7,8 & 3,99 \\
\hline 6 & 2,273 & 0,848 & 1,68 & 63 & 5 & 44 & 111 & 164 & 7,1 & 3,57 \\
\hline 7 & 2,171 & 0,803 & 1,71 & 63 & 4 & 42 & 134 & 168 & 6,8 & 3,2 \\
\hline 8 & 1,754 & 0,911 & 0,92 & 48 & 5 & 43 & 90 & 113 & 8,1 & 2,51 \\
\hline 9 & 2,201 & 0,868 & 1,53 & 60 & 5 & 44 & 109 & 126 & 9,2 & 3,76 \\
\hline 10 & 2,221 & 0,923 & 1,41 & 58 & 7 & 47 & 116 & 145 & 6,4 & 4,24 \\
\hline 11 & 2,381 & 0,957 & 1,48 & 60 & 7 & 52 & 134 & 123 & 5,6 & 4,32 \\
\hline 12 & 2,351 & 0,893 & 1,63 & 61 & 6 & 45 & 121 & 158 & 7,9 & 3,89 \\
\hline 13 & 2,231 & 0,973 & 1,29 & 56 & 7 & 47 & 122 & 135 & 9,7 & 4,05 \\
\hline 14 & 2,251 & 0,972 & 1,31 & 57 & 7 & 42 & 124 & 152 & 7,1 & 4,16 \\
\hline
\end{tabular}




\subsection{Caracterización mineralógica.}

La determinación de los minerales constituyentes de las muestras de diatomita se ha realizado mediante microscopía óptica, difracción de rayos $\mathrm{X}$ y análisis térmico diferencial (Termobalanza Chyo TRDA-3H).

\section{MICROSCOPÍA ÓPTICA.}

Se observa sobre todo la presencia de sílice amorfa, con algunos restos de cuarzo y fragmentos de conchas o esqueletos; también se identifica circón, así como óxidos de hierro, y un cemento muy blando, constituido en parte por material arcilloso fino.

\section{DIFRACCIÓN DE RAYOS X.}

Los difractogramas sobre muestras molidas se han obtenido con cátodo de cobalto, utilizando un equipo de difracción Philips PW, modelo 1965/40. Las condiciones de trabajo fueron $30 \mathrm{~mA}, 40 \mathrm{kV}$ y una velocidad de goniómetro de 2 grados por minuto, desde 2 hasta 80 grados, encontrándose los picos más representativos entre los 15 y los 40 grados.

En la figura 2 se muestran los difractogramas tipo de las muestras estudiadas. En ellos se revela la existencia de las siguientes especies minerales: cuarzo (Q), cristobalita (Crist), carbonato cálcico (Ca), ortosa (Ort) y plagioclasas (Plg).

Si bien la sílice existente en las muestras se puede encontrar como sílice amorfa, cuarzo y cristobalita, por tratarse de muestras constituidas básicamente por sílice amorfa, los difractogramas indican una baja cristalinidad en la región de la difracción principal de la cristobalita. La presencia de ortosa y plagioclasas se correlaciona con los valores importantes de óxido de aluminio, sodio y potasio, que revelan los análisis químicos de las muestras estudiadas.

La calcita se observa en todos los difractogramas, siendo más abundante en las muestras M-7 y M-8.

\section{ANÁLISIS TÉRMICO-DIFERENCIAL.}

Se utilizó una termobalanza Chyo TRDA-3H, de análisis termodiferencial (ATD) y termogravimétrico (ATG) simultáneos, con el siguiente grado de sensibilidad para los registros:

$$
\text { ATD: } 10 \mu \mathrm{V}-1.000 \mu \mathrm{V} \text {. }
$$

ATG: 50mg - 1.000mg.

En la figura 3 a y 3 b se representan las curvas de ATD y ATG de las muestras estudiadas en atmósfera de nitrógeno. Estudiadas las curvas se pueden observar las siguientes reacciones:

- En las curvas de ATD, aparece entre 100 y $500^{\circ} \mathrm{C}$, un ligero efecto endotérmico, el cual está acompañado de la correspondiente perdida de peso en la curva de ATG.

- En las curvas de ATD de las muestras M-7 y M-8 se observa además, un efecto endotérmico entre 550 y $850^{\circ} \mathrm{C}$, debido a la descomposición del carbonato cálcico, junto al que aparece la correspondiente pérdida de peso en la curva de ATG.

- Las reacciones que se deducen de las distintas curvas son muy similares en todas las muestras, siendo la pérdida de peso promedio del $6,59 \%$, la cual no es una cifra muy significativa. Este comportamiento se justifica por la pérdida del agua de constitución de la sílice, que tiene lugar entre los $100 \mathrm{y} \operatorname{los} 550^{\circ} \mathrm{C}$, y del escaso material arcilloso presente en cada una de las muestras estudiadas, el cual pierde su agua de constitución entre los 200 y los $500^{\circ} \mathrm{C}$.

- De entre todas las muestras estudiadas, las M-11, M-13 y M14 son las que presentan un comportamiento térmico más moderado, con pérdidas de peso inferiores a 1,70 \% y ausencia de grandes efectos en la curva de ATD. En general, y dado este favorable comportamiento térmico, cabe pensar que la diatomita de Río Seco sería un material de características idóneas para su empleo como materia prima en la industria cerámica.

TABLA II.

ANÁlisis QUímico de LAS DiATOMITAS DE "Río SECO".

\begin{tabular}{|c|c|c|c|c|c|c|c|c|c|c|c|}
\hline MUESTRAS & $\mathrm{S} 102$ & $\mathrm{TiO} 2$ & A1203 & $\mathrm{Fe203}$ & $\mathrm{MgO}$ & $\mathrm{MnO}$ & $\mathrm{CaO}$ & $\mathrm{Na} 2 \mathrm{O}$ & $\mathrm{K} 2 \mathrm{O}$ & P205 & $P \times C$ \\
\hline$M-1$ & 63,9 & 0,29 & 14,4 & 2,00 & 0,87 & 0,04 & 1,76 & 3,64 & 3,21 & 0,06 & 9,38 \\
\hline$M-2$ & 64,1 & 0,31 & 14,5 & 2,11 & 0,88 & 0,04 & 1,76 & 3,52 & 3,23 & 0,06 & 9,34 \\
\hline$M-3$ & 66,6 & 0,28 & 13,8 & 1,94 & 0,68 & 0,05 & 1,92 & 3,44 & 3,51 & 0,06 & 7,32 \\
\hline$M-4$ & 66,9 & 0,28 & 13,7 & 1,94 & 0,71 & 0,05 & 1,89 & 3,53 & 3,54 & 0,06 & 7,27 \\
\hline M-S & $\infty, 2$ & 0,27 & 13,6 & 1,91 & 0,64 & 0,05 & 1,51 & 3,51 & 3,30 & 0,06 & 6,63 \\
\hline$M-6$ & 67,9 & 0,27 & 13,5 & 1,88 & 0,64 & 0,05 & 1,51 & 3,65 & 3,31 & 0,05 & 6,63 \\
\hline$M-7$ & 61,4 & 0,22 & 12,8 & 1,51 & 0,82 & 0,04 & 8,77 & 3,11 & 3,05 & 0,07 & 6,61 \\
\hline MO & 6,2 & 0,27 & 12,1 & 1,87 & 0,63 & 0,05 & 1,52 & 3,74 & 3,28 & 0,05 & 6,62 \\
\hline M-10 & 65,8 & 0,24 & 12,1 & 1,69 & 0,88 & 0,04 & 1,71 & 2,88 & 2,55 & 0,06 & 9,61 \\
\hline M-11 & 72,1 & 0,31 & 14,3 & 2,00 & 0,81 & 0,05 & 1,91 & 3,92 & 3,26 & 0,07 & 1,68 \\
\hline$M-12$ & 60,1 & 0,18 & 12,8 & 1,49 & 1,29 & 0,04 & 1,97 & 1,78 & 2,15 & 0,07 & 8,87 \\
\hline$M-13$ & 72,1 & 0,22 & 14,6 & 1,49 & 0,83 & 0,06 & 1,71 & 3,18 & 3,04 & 0,07 & 1,53 \\
\hline M-14 & 72,2 & 0,29 & 14,3 & 1,91 & 1,09 & 0,04 & 1,81 & 3,48 & 3,21 & 0,06 & 1,37 \\
\hline
\end{tabular}




\section{CONCLUSIONES.}

Las diatomitas del yacimiento de "Río Seco" presentan unos contenidos medios en sílice de $66,9 \%$, valores bastante bajos con respecto a los valores de referencia de la diatomita natural Foselite-24. Este valor en sílice, se puede considerar en cierto modo como un índice de la calidad de la diatomita. Las impurezas, están representadas fundamentalmente por óxidos de hierro y aluminio, los cuales alcanzan unos valores promedio de $1,84 \%$ y $13,5 \%$ respectivamente.

Para la mayor parte de las aplicaciones industriales de la diatomita, además de elevados contenidos en sílice, se precisa

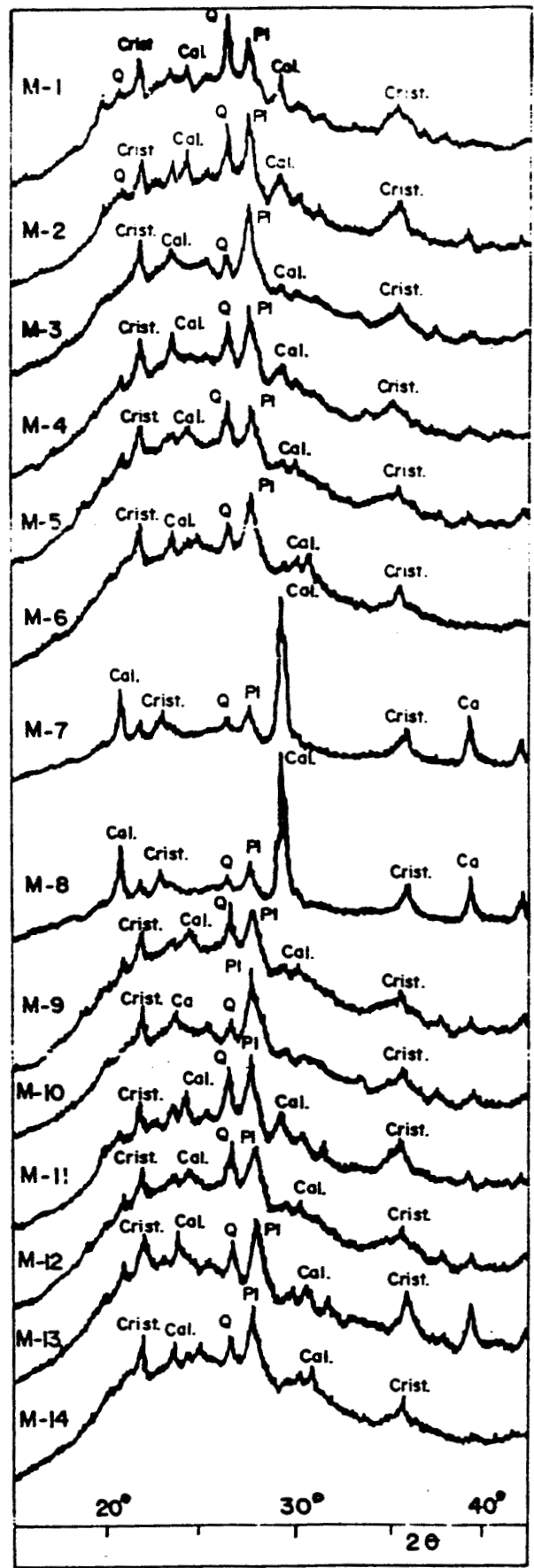

Co = Colcito

Crist. = Cristobaitito

PI = Plegiocloso

0 = Cuarzo

Fig. 2. Difractograma de 14 muestras de diatomitas de "Río Seco".

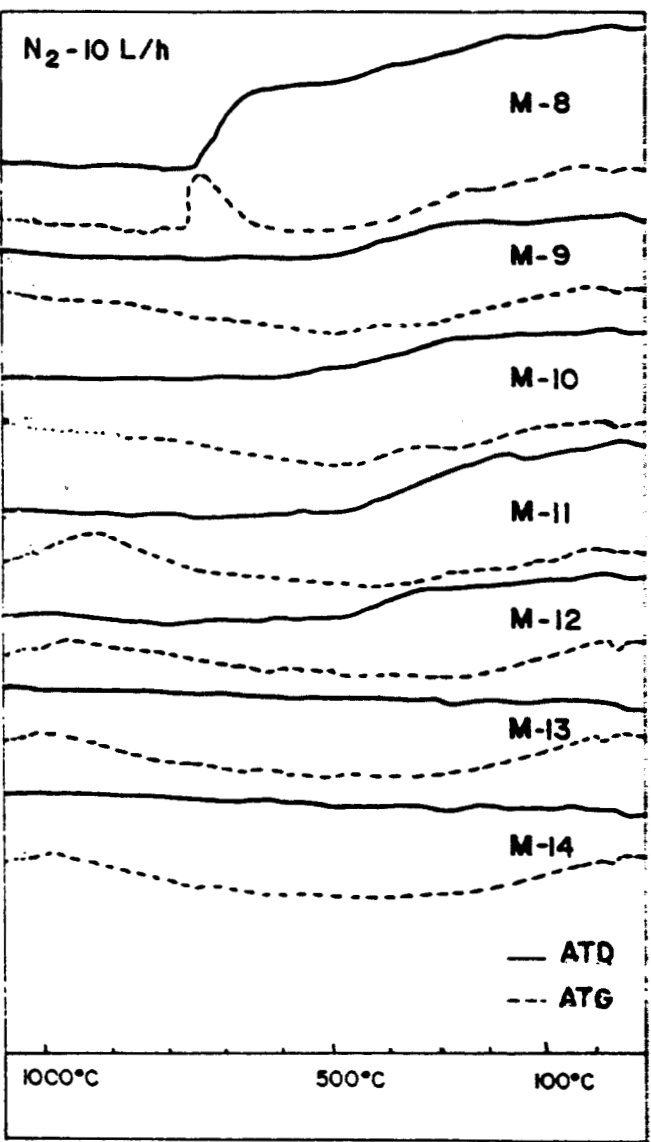

Fig. 3 a. Diagramas de ATD y ATG de las muestras M-1 a M-7.

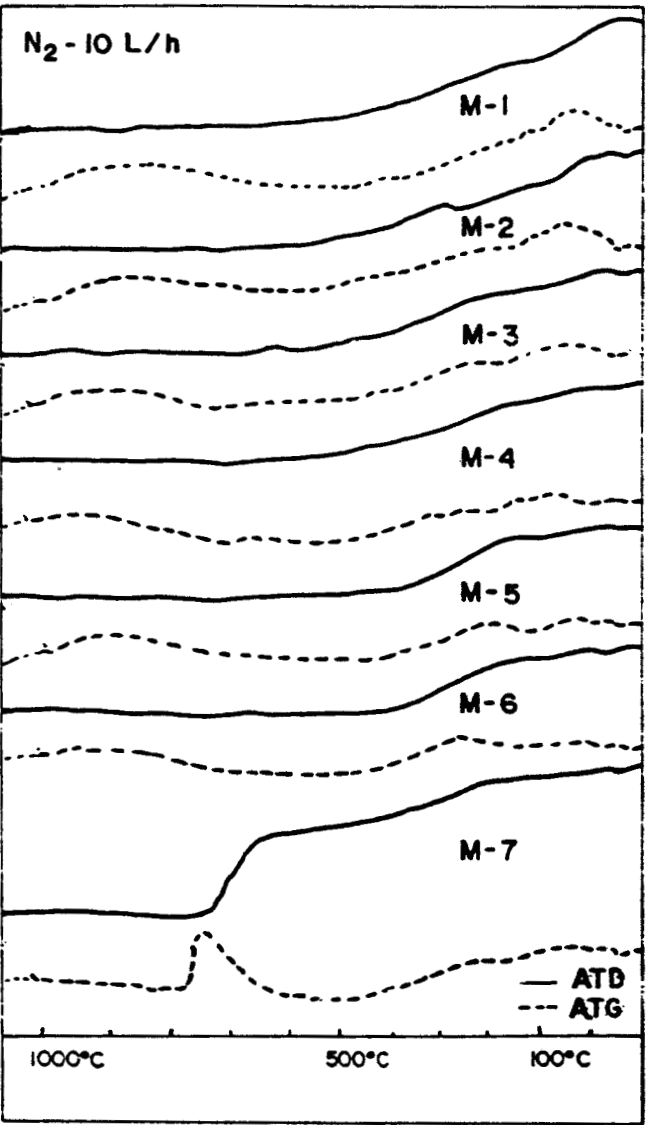

Fig. 3 b. Diagramas de ATD y ATG de las muestras M-8 a M-14. 
un material en el cual los porcentajes de contaminantes, considerando como tales a los componentes no silíceos de la misma, sean los menores posibles (12). En este sentido, las diatomitas del yacimiento de "Río Seco" presentan contenidos importantes de impurezas, que habría que eliminar mediante un tratamiento de lavado previo, para así alcanzar las especificaciones comerciales requeridas.

Por tanto, de acuerdo con sus características físico-químicas, estas diatomitas se pueden considerar de calidad media, en las que los porcentajes de sílice y de alúmina que presentan, aconsejarían derivar sus aplicaciones hacia campos en los que pueda resultar útil, bien como material puzolánico, o bien en la fabricación de materiales cerámicos para construcción. Tampoco deben olvidarse sus posibles aplicaciones como material aislante térmico y acústico.

\section{AGRADECIMIENTOS}

Los autores expresan su agradecimiento a la agencia española de Cooperación Internacional (AECI), a la Oficina de Cooperación con Iberoamérica de la Universidad de Oviedo (España), y a todas aquellas Universidades e Instituciones peruanas relacionadas con el trabajo.

\section{BIBLIOGRAFÍA.}

L.F.Verdeja, J:P: Sancho, J:L: Barranzuela, E.R. Vasquez. “Características físicoquímicas de las diatomitas de Bayovar (Perú)".Bol. Soc. Esp. Cerámica y Vidrio. 29 (2), 87-93.1990.

L.F. Verdeja, J.L. Barranzuela, J.P. Sancho, J.M. Ayala, E.R. Vasquez. "Características refractarias de las diatomitas de Bayovar. Perú". Congreso IBEROMET-CONAMET VI, Santiago de Chile, Tomo I, 589-601. 1990.

L.F. Verdeja, J.M. Ayala, M.A. Llavona, E.R. Vasquez, J.L. Barranzuela. “Las diatomitas del Perú". Revista de Minas. 8, 121-129. 1993.

J.L. Vega, J. García Iglesias, L.F. Verdeja. “Los recursos mineros de la región Grau (Perú)". Rocas y Minerales, n¹1, 28-34. 1993.

D.L. Durham. “Diatomite”. U.S. Mineral Ressources. U.S. Geol. Prof. Paper, 820, 191-195. 1973.

A.C. Meisinger. “Diatomite, mineral facts and problems”. Bull. 671. 1-7. 1980.

J.P. Calvo Sorando. Diatomitas: Geología y usos". En: Yacimientos minerales. 635-643.

F.L. Kadey. "Industrial minerals and rocks". Am. Inst. Mining Metallurgical and Petroleum Engs. Inc. 605-635. 1975.

B.J. Agramonte. "Las diatomitas de Ayacucho y su paralelo con las de Tarucani y Bayovar". Bol. Soc. Geol. Perú. 72, 259-269. 1983.

G.H. McDonald. "Miocene of the Sechura desert, Piura”. Bol. Soc. Geol. Perú. 30, 225-242. 1956.

Instituto Geológico Minero Metalúrgico Perú. “Inventario nacional de sustancias no metálicas". Lima. 1982.

K. Harries-Rees. "Diatomite: Stability under threat". Industrial minerals and rocks, April, 33-43. 1994.

J.R. Rodríguez Lichtenheldt. "Investigación de las diatomitas de Río Seco y sus aplicaciones industriales". Tesis Doctoral. Univ. Oviedo. 292p. 1996.

\section{CONFERENCIA HISPANO-FRANCESA SOBRE LAS APLICACIONES PIEZOELECTRICAS}

\section{Objetivos:}

El objetivo de esta conferencia es el intercambio de los últimos conocimientos sobre los materiales piezoeléctricos y sus aplicaciones, siendo de interés para las comunidades científica e industrial.

\section{Inscripciones:}

Las inscripciones a la conferencia son:

Tarifa normal: 25.000 pts.

Tarifa estudiante: 12.500 pts.

La inscripción incluye las actas de la conferencia y las comidas de los días 29 y 30 de Octubre

\section{Para más información:}

Silvia Soriano. CAP'98

Departmament de Física Aplicada. Campus Nord. Mod. B5.

C/ Jordi Girona 1-3

08034 Barcelona

Tel: 934016802

Fax: 934016802

Email: silvia@benard.upc.es

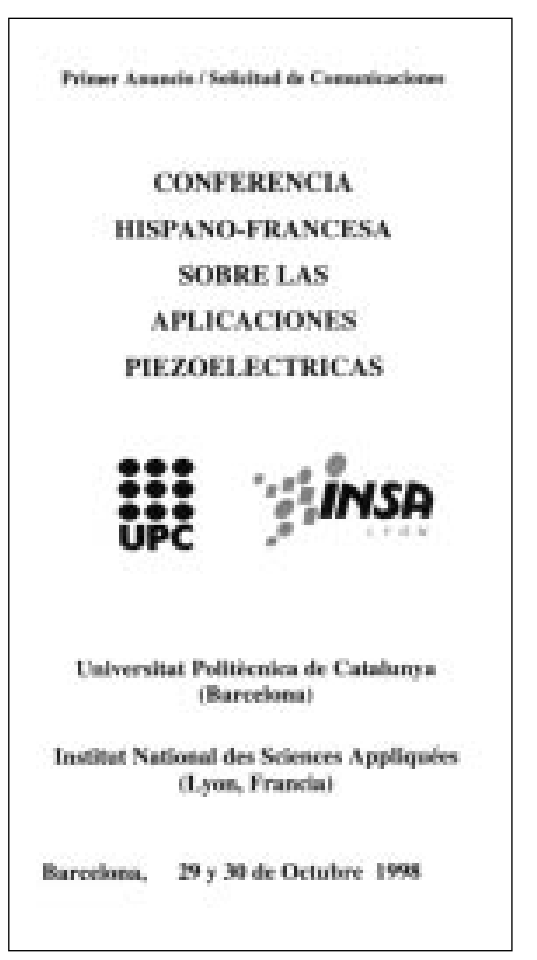

